\title{
Solving multiple linear regression problem using artificial neural network
}

\author{
Mohammad S. Khrisat, Ziad A. Alqadi \\ Computer Engineering Department, Faculty of Engineering Technology, Applied University, Amman, Jordan
}

\begin{tabular}{lll}
\hline \hline Article Info & ABSTRACT \\
\cline { 1 - 2 } Article history: & $\begin{array}{l}\text { Multiple linear regressions are an important tool used to find the relationship } \\
\text { between a set of variables used in various scientific experiments. In this } \\
\text { article we are going to introduce a simple method of solving a multiple } \\
\text { Received Nov 14, 2020 }\end{array}$ & $\begin{array}{l}\text { rectilinear regressions (MLR) problem that uses an artificial neural network } \\
\text { to find the accurate and expected output from MLR problem. Different } \\
\text { artificial neural network (ANN) types with different architecture will be } \\
\text { Accepted Jul 26, 2021 }\end{array}$ \\
$\begin{array}{l}\text { tested, the error between the target outputs and the calculated ANN outputs } \\
\text { will be investigated. A recommendation of using a certain type of ANN } \\
\text { based on the experimental results will be raised. }\end{array}$ \\
Artificial neural network
\end{tabular}

Convolutional artificial neural

network

Feedforward artificial neural

network

This is an open access article under the CC BY-SA license.

Multiple linear regression

\section{Corresponding Author:}

Mohammad S. Khrisat

Computer Engineering Department, Faculty of Engineering Technology, Applied University

Amman 11134, P.O. Box 15008 Jordan

Email: mkhrisat@bau.edu.jo

\section{INTRODUCTION}

Multiple rectilinear regressions (MLR) [1] are the foremost common kind of linear regression analysis. As a prognostic analysis, it will not justify the link between one continuous dependent variable and 2 or a lot of freelance variables. The independent variables will be continuous or categorical (dummy coded as appropriate). MLR presented in (1) and Figure 1 can be solved using MATLAB Figure 2 [2]-[4].

$$
y=a_{0}+a_{1} x_{1}+a_{2} x_{2}+\cdots+a_{n} x_{n}
$$

Where:

$$
\begin{aligned}
& \mathrm{y}=\text { dependent variable } \\
& \mathrm{x}=\text { explanatory variables } \\
& \mathrm{a}=\text { constants }
\end{aligned}
$$

The coefficients will be as follows: $a=\{5.0,2.0,3.5\}$, So MLR problem can be represented using (2).

$$
y=5+2 x_{1}+3.5 x_{2}
$$

Artificial neural network (ANN) [5]-[7] is a computational mathematical model, which contains a set of fully connected neurons, which are arranged in two or more layers. Each neuron as shown in Figure 3 implements two operations [8]-[10]: i) finding the sum of products of the inputs and the associated weights [11], [12], ii) according to the selected activation function calculate the neuron output. 


\begin{tabular}{rrc} 
X1 & \multicolumn{1}{c}{ X2 } & Target output \\
2.0000 & -3.0000 & -1.5000 \\
3.0000 & -4.5000 & -4.7500 \\
-2.0000 & -25.0000 & -86.5000 \\
4.0000 & 0 & 13.0000 \\
-5.0000 & 9.5000 & 28.2500 \\
0 & 10.0000 & 40.0000 \\
9.0000 & 11.0000 & 61.5000 \\
10.0000 & 2.0000 & 32.0000 \\
15.0000 & 3.0000 & 45.5000 \\
-11.0000 & 12.0000 & 25.0000 \\
12.5000 & -8.0000 & 2.0000 \\
18.0000 & -2.0000 & 34.0000 \\
22.3000 & 0 & 49.6000 \\
4.0000 & 14.0000 & 62.0000 \\
0 & 5.0000 & 22.5000 \\
-6.8000 & 11.0000 & 29.9000 \\
\hline
\end{tabular}

Figure 1. MLR example

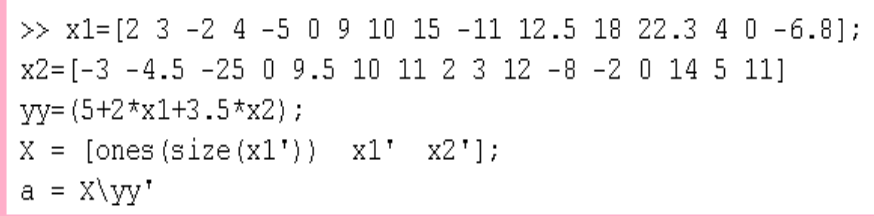

Figure 2. MATLAB code to solve MLR

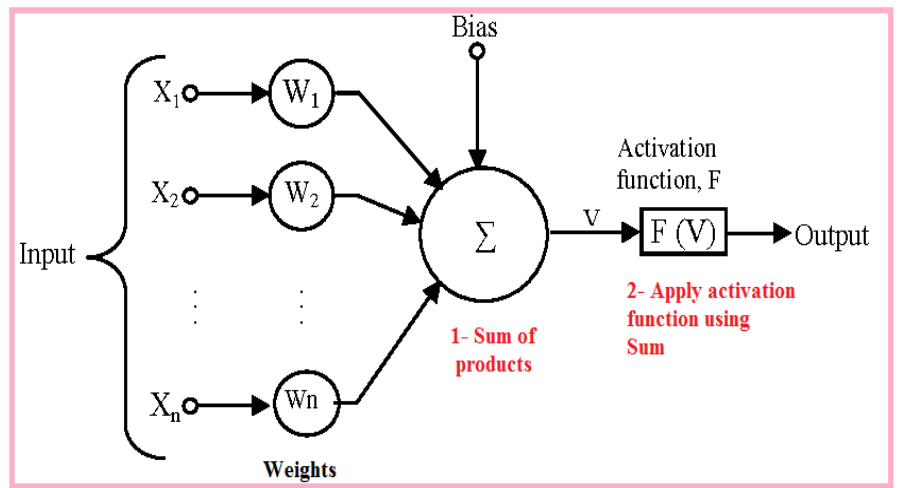

Figure 3. Neuron operations [13], [14]

Different types of ANN can be used to solve MLR problem, the first one is feed forward ANN with back propagation (FFANN), in this ANN as shown in Figure 4 each neuron in any layer are fully connected to the previous inputs [15]-[17]. The second type is cascade feed forward ANN (CFANN) [18], [19], in this type of ANN the inputs are used to feed the hidden layer as shown in Figure 5. The last type which will be investigated in this paper is Elman ANN (EANN), in this type as shown in Figure 6 a context inputs formed from the hidden layer are used as inputs [20], [21].

To use any type of ANN to solve MLR problem we have to follow the steps [22]-[24]: i) select the input data set, which is the values of explanatory variables; ii) select the target, which is the values of dependent variable; iii) create ANN, here we define the type of ANN, ANN architecture: How many layers are to be used, and how many neurons in each layer. In this step we have to define activation function for each layer, logsig or tansig for the input layer and the hidden layers, linear activation function for the output layer [25]; iv) initialize the weights; v) set the error parameter to zero; vi) select the number of training cycles; vii) train ANN; viii) save ANN, ix) run ANN to solve MLR problem. 


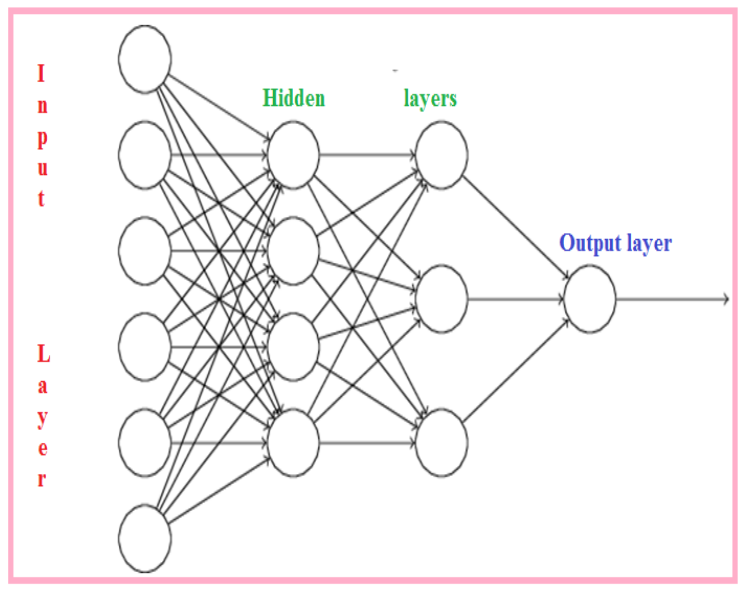

Figure 4. FFANN

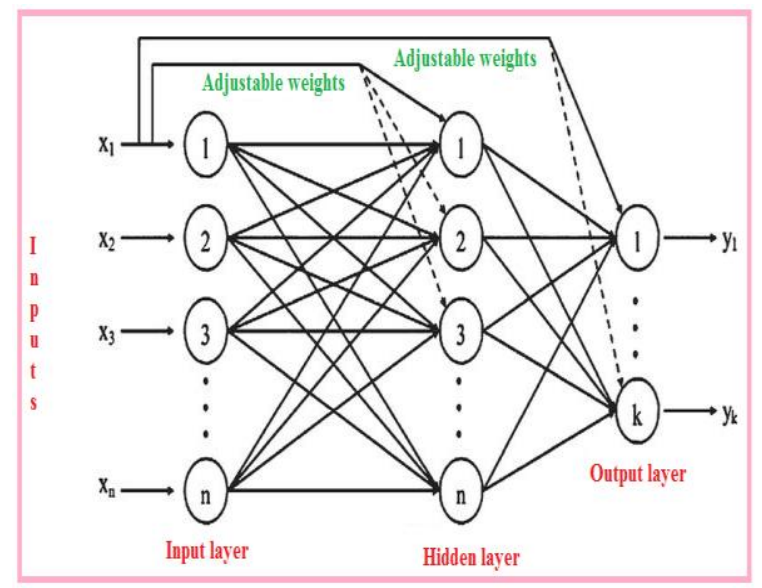

Figure 5. CFANN

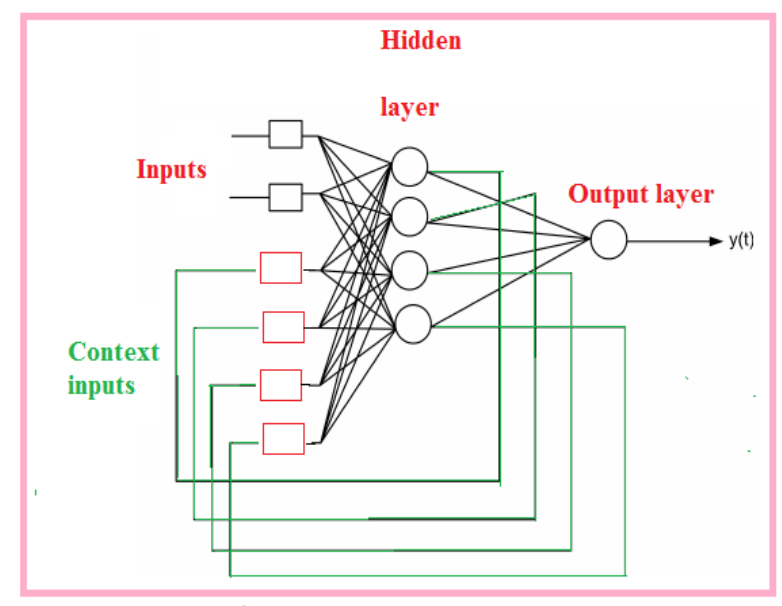

Figure 6. EANN

\section{RESEARCH METHOD}

Different types of ANN [11]-[13] with different architectures were used to solve MLR problem, some experiments were tested, and below we will investigate the results of these experiments:

Experiment 1:

Here we select a two layers ANN, input layer with two neurons and output layer with one neuron, Table 1 shows the obtained results of using various types of ANN: maximum error was calculated using (3).

$$
\text { MaxErr }=\max \left(\operatorname{abs}\left(y_{1}-y\right)\right)
$$

Figure 7 shows the obtained outputs for various ANN. Figures 8, 9, and 10 shows the error between the target and the calculated output of each ANN. From the obtained results here in this experiment we can see that CFANN has the best performance and we can recommend it to solve MLR problem.

Table 1. Experiment 1 results

\begin{tabular}{cccccc}
\hline ANN type & Number of layers & Neurons & Activation function & Max. Error & Training cycles \\
\hline FFANN & 2 & 2,1 & Logsig, linear & 0.0079 & 10000 \\
CFANN & $\mathbf{2}$ & $\mathbf{2 , 1}$ & Logsig, linear & $\mathbf{0}$ & $\mathbf{9}$ \\
EANN & 2 & 2,1 & Logsig, linear & 31.1152 & 10000 \\
FFANN & 2 & 2,1 & Tansig, linear & 0.0001059 & 10000 \\
CFANN & $\mathbf{2}$ & $\mathbf{2 , 1}$ & Tansig, linear & $\mathbf{0}$ & $\mathbf{1 0}$ \\
EANN & 2 & 2,1 & Tansig, linear & 24.7341 & 10000 \\
\hline
\end{tabular}




\begin{tabular}{|crrr|}
\hline Target & CF output & FF output & ELM output \\
-1.5000 & -1.5000 & -1.5000 & -2.1458 \\
-4.7500 & -4.7500 & -4.7500 & -5.2117 \\
-86.5000 & -86.5000 & -86.5000 & -61.0822 \\
13.0000 & 13.0000 & 13.0000 & 14.9681 \\
28.2500 & 28.2500 & 28.2500 & 42.5609 \\
40.0000 & 40.0000 & 40.0000 & 47.1758 \\
61.5000 & 61.5000 & 61.5000 & 50.1927 \\
32.0000 & 32.0000 & 32.0000 & 37.1704 \\
45.5000 & 45.5000 & 45.5000 & 45.3666 \\
25.0000 & 25.0000 & 25.0000 & -5.3184 \\
2.0000 & 2.0000 & 2.0000 & -4.7442 \\
34.0000 & 34.0000 & 34.0000 & 33.4190 \\
49.6000 & 49.6000 & 49.6000 & 45.0598 \\
62.0000 & 62.0000 & 62.0000 & 50.4441 \\
22.5000 & 22.5000 & 22.5000 & 33.4453 \\
29.9000 & 29.9000 & 29.9000 & 29.3088 \\
\hline
\end{tabular}

Figure 7. Obtained outputs for each ANN

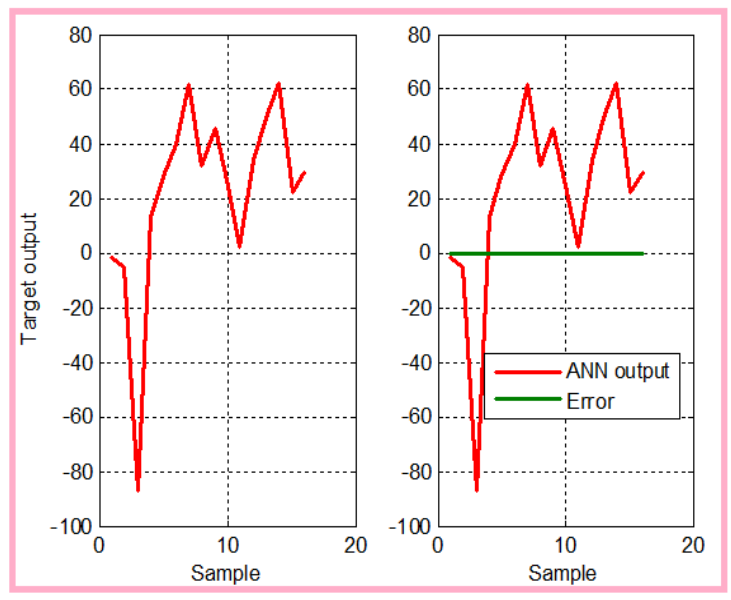

Figure 9. Calculated error for FFANN

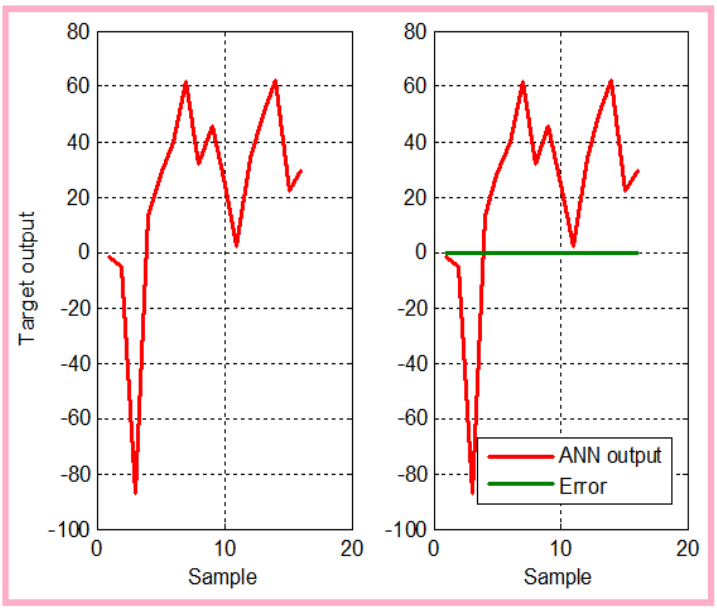

Figure 8. Calculated error for CFANN

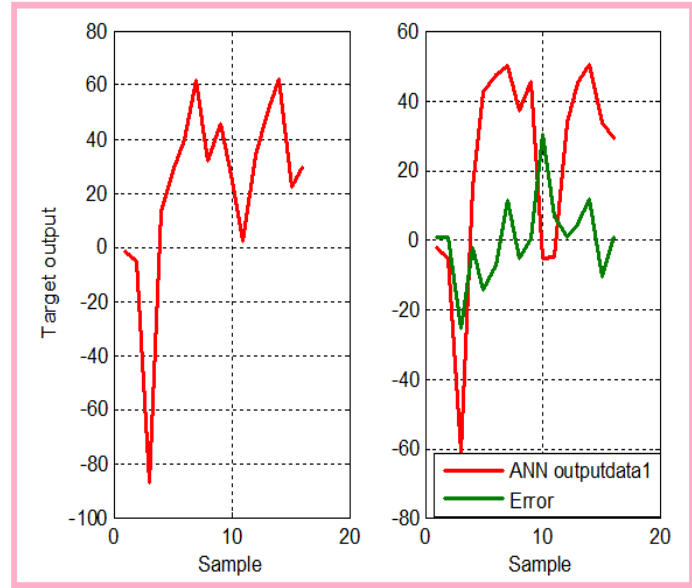

Figure 10. Calculated error for EANN

Experiment 2:

The architectures of ANNs here were changed to see how changing ANN architecture will affect the performance, Table 2 shows the experimental results. From Table 2 we can see that changing ANN architecture does not positively affect the performance; hence we can conclude that using CFANN with two layers is best solution of MLR problem, here the number of neurons in the input layer must equal to the number of explanatory variables. Figure 11 shows the calculated outputs of using CFANN to solve MLR problem with 4 explanatory variables (here the number of neurons in the input layer 4), while Figure 12 shows the error between the target and the calculated output.

Table 2. Experiment 2 results

\begin{tabular}{cccccc}
\hline ANN type & Number of layers & Neurons & Activation function & Max. Error & Training cycles \\
\hline FFANN & 3 & $2,4,1$ & Tansig, tansig, linear & 0.00014588 & 10000 \\
CFANN & $\mathbf{3}$ & $\mathbf{2 , 4 , 1}$ & Tansig, tansig, linear & $\mathbf{0}$ & $\mathbf{7 9 6}$ \\
EANN & 3 & $2,4,1$ & Tansig, tansig, linear & 55.5833 & 10000 \\
FFANN & 3 & $2,4,1$ & logsig, tansig, linear & 0.00013311 & 10000 \\
CFANN & $\mathbf{3}$ & $\mathbf{2 , 4 , 1}$ & logsig, tansig, linear & $\mathbf{0}$ & $\mathbf{1 1 7 1}$ \\
EANN & 3 & $2,4,1$ & logsig, tansig, linear & 25.0369 & 10000 \\
FFANN & 3 & $2,4,1$ & logsig, tansig, linear & 0.0048 & 10000 \\
CFANN & $\mathbf{3}$ & $\mathbf{2 , 4 , 1}$ & logsig, tansig, linear & $\mathbf{0}$ & $\mathbf{1 1 4}$ \\
EANN & 3 & $2,4,1$ & logsig, tansig, linear & 58.0207 & 10000 \\
FFANN & 3 & $2,4,1$ & Tansig, logsig, linear & 0.0034 & 10000 \\
CFANN & $\mathbf{3}$ & $\mathbf{2 , 4 , 1}$ & Tansig, logsig, linear & $\mathbf{0}$ & $\mathbf{3 1 7}$ \\
EANN & 3 & $2,4,1$ & Tansig, logsig, linear & 29.515 & 10000 \\
\hline
\end{tabular}




\begin{tabular}{|rrrccc|}
\hline $\mathbf{X} 1$ & $\mathbf{X} 2$ & $\mathbf{X} 3$ & $\mathbf{X} 4$ & Target & CFANN 0utput \\
2.0000 & -3.0000 & 1.0000 & -1.5000 & 4.3000 & 4.3000 \\
3.0000 & -4.5000 & 1.5000 & -3.0000 & 4.8500 & 4.8500 \\
-2.0000 & -25.0000 & -1.0000 & -23.5000 & -62.3000 & -62.3000 \\
4.0000 & 0 & 2.0000 & 1.5000 & 19.2000 & 19.2000 \\
-5.0000 & 9.5000 & -2.5000 & 11.0000 & 5.0500 & 5.0500 \\
0 & 10.0000 & 0 & 11.5000 & 26.2000 & 26.2000 \\
9.0000 & 11.0000 & 4.5000 & 12.5000 & 64.5000 & 64.5000 \\
10.0000 & 2.0000 & 5.0000 & 3.5000 & 47.8000 & 47.8000 \\
15.0000 & 3.0000 & 7.5000 & 4.5000 & 70.1000 & 70.1000 \\
-11.0000 & 12.0000 & -5.5000 & 13.5000 & -13.2000 & -13.2000 \\
12.5000 & -8.0000 & 6.2500 & -6.5000 & 34.8000 & 34.8000 \\
18.0000 & -2.0000 & 9.0000 & -0.5000 & 70.6000 & 70.6000 \\
22.3000 & 0 & 11.1500 & 1.5000 & 92.4000 & 92.4000 \\
4.0000 & 14.0000 & 2.0000 & 15.5000 & 51.4000 & 51.4000 \\
0 & 5.0000 & 0 & 6.5000 & 14.7000 & 14.7000 \\
-6.8000 & 11.0000 & -3.4000 & 12.5000 & 1.3000 & 1.3000 \\
\hline
\end{tabular}

Figure 11. Results of MLR with 4 variables

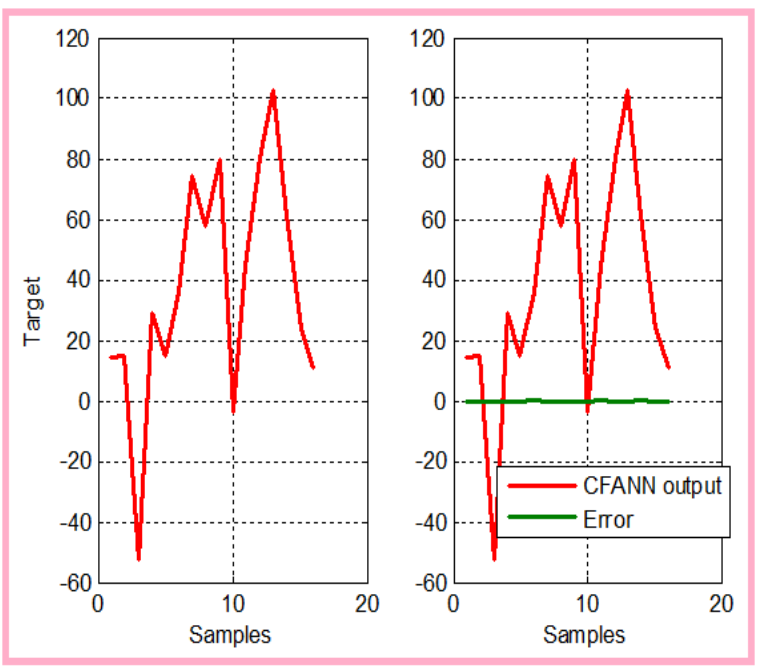

Figure 12. Calculated error for CFANN

\section{CONCLUSION}

Different types of ANNs with different architectures were used to solve MLR problem. Each ANN was treated with various input data set varying the activation function. It was seen that CFANN has the best performance, and here we can highly recommend CFANN to solve MLR problem with any number of explanatory variables by selecting two layers and adjusting the number of neurons in the input layer to match the number of explanatory variables in MLR problem. Provide a statement that what is expected, as stated in the "Introduction" chapter can ultimately result in "Results and Discussion" chapter, so there is compatibility. Moreover, it can also be added the prospect of the development of research results and application prospects of further studies into the next (based on result and discussion).

\section{REFERENCES}

[1] K. L. L. Khine and T. T. S. Nyunt, "Predictive geospatial analytics using principal component regression," International Journal of Electrical and Computer Engineering (IJECE), vol. 10, no. 3, pp. 2651-2658, 2020, doi: 10.11591/ijece.v10i3.pp2651-2658.

[2] M. S. Rodrigo, "A system based on artificial neural networks for automatic classification of hydro-generator stator windings partial discharges," Journal of Microwaves, Optoelectronics and Electromagnetic Applications, vol. 16, no. 3, pp. 628-645, 2017, doi: 10.1590/2179-10742017v16i3854

[3] M. Linveh and I. Ishai, "Using indicative properties to predict the density-moisture relationship of soil," Transportation Research Record, vol. 60P, pp. 22-28, 1978. 
[4] N. Iksan, J. Sembiring, N. Hariyanto, and S. H. Supangkat, "Residential load event detection in NILM using robust cepstrum smoothing based method," International Journal of Electrical and Computer Engineering (IJECE), vol. 9, no. 2, pp. 742-752, 2019, doi: 10.11591/ijece.v9i2.pp742-752.

[5] F. Recknagela, M. Frenchb, and P. H. K. I. Yabunakad, "Artificial neural network approach for modelling and prediction of algal blooms," Ecological Modelling, vol. 96, no. 1-3, pp. 11-28, 1997, doi: 10.1016/S0304-3800(96)00049-X.

[6] Z. A. AlQadi, A. Y. Hindi, and O. D. Majed, "Procedures for speech recognition using LPC and ANN," International Journal of Engineering Technology Research \& Management, vol. 4, no. 2, pp. 48-55, 2020.

[7] Z. Alqadi, M. S. Khrisat, Y. Eltous, S. A. Khawatreh, and M. O. Dwairi, "Building face recognition system (FRS)," International Journal of Computer Science and Mobile Computing, vol. 9, no. 6, pp. 15-24, 2020.

[8] J. Al-Azzeh N. Asad, Z. Alqadi, I. Shayeb, and Q. Jaber, "Simple procedures to create HSCS," International Journal of Engineering Research and Management (IJERM), vol. 7, no. 5, pp. 6-10, 2020.

[9] DA. Hindi, M. O. Dwairi, and Z. Alqadi, "Analysis of procedures used to build an optimal fingerprint recognition system," International Journal of Computer Science and Mobile Computing, vol. 9, no. 2, pp. 21-37, 2020.

[10] H. E. Dytch and G. L. Wied, "Artificial neural networks and their use in quantitative pathology," Analytical and Quantitative Cytology and Histology, vol. 12, no. 6, pp. 379-393, 1990.

[11] M. A. Mohamad, H. Haron, and N. A. Ali, "Prediction of hardness in titanium aluminium nitride TiA1N coating process: A review," 2012 Fourth International Conference on Computational Intelligence, Modelling and Simulation, pp. 111-116, 2012, doi: 10.1109/CIMSim.2012.63.

[12] U. Bai, Y. Li, Y. Liu, and Z. Ma, "Short-term prediction of distribution network faults based on support vector machine," 2017 12th IEEE Conference on Industrial Electronics and Applications (ICIEA), 2017, pp. 1421-1426, doi: 10.1109/ICIEA.2017.8283062.

[13] H. Yi, D. Ding, M. Lu, and Li. Li, "Fault location based on relevance vector machine and decision directed acyclic graph," 2013 25th Chinese Control and Decision Conference (CCDC), 2013, pp. 4679-4684, doi: 10.1109/CCDC.2013.6561780.

[14] Y. Temprado, F. J. Molinero, C. Garcia, and J. Gomez, "Knowledge discovery from trouble ticketing reports in a large telecommunication company," 2008 International Conference on Computational Intelligence for Modelling Control \& Automation, 2008, pp. 37-42, doi: 10.1109/CIMCA.2008.116.

[15] P. Kayal, S. Chanda, and C. K. Chanda, "An ANN based network reconfiguration approach for voltage stability improvement of distribution network," 2011 International Conference on Power and Energy Systems, 2011, pp. 1-7, doi: 10.1109/ICPES.2011.6156643.

[16] F. J. Anscombe and J. W. Tukey, "The examination and analysis of residuals" Technometrics, vol. 5, no. 2, pp. 141-160, 1963, doi: $10.2307 / 1266059$.

[17] R. A. Becker, J. M. Chambers, and A. R. Wilks, "The new S language a programming environment for data analysis and graphics," Pacific Grove, CA: Wadsworth and Brooks/Cole, 1988.

[18] J. Chang and D. J. Olive, "OLS for 1D regression models," Communications in statistics: Theory and methods, vol. 39, no. 10, 1869-1882, 2010, doi: 10.1080/03610920902923494.

[19] R. D. Cook, "Regression graphics: Ideas for studying regression through graphics," New York, NY: Wiley, 1998.

[20] S. Ghosh, "Note on a common error in regression diagnostics using residual plots," The American Statistician, vol. 41, pp. 338, 1987.

[21] H. L. Harter, "The method of least squares and some alternatives: Part IV," International Statistical Review, vol. 43, no. 2, pp. 125-190, 1975, doi: 10.2307/1402897.

[22] G. Ames, D. Witten, T. Hastie, and R. Tibshirani, "An introduction to statistical learning with applications," R. New York, NY: Springer, vol. 103, 2013.

[23] J. Lei and L. Wasserman, "Distribution free prediction bands for non-parametric regression," Journal of the Royal Statistical Society: Series B: Statistical Methodology, vol. 76, no. 1, pp. 71-96, 2014.

[24] R. Bandi, J. Amudhavel, and R. Karthik, "Machine learning with pyspark-review," Indonesian Journal of Electrical Engineering and Computer Science (IJEECS), vol. 12, no. 1, pp. 102-106, 2018, doi: 10.11591/ijeecs.v12.i1.pp102-106.

[25] L. S. Mezher, "Design and implementation hamming neural network with VHDL," Indonesian Journal of Electrical Engineering and Computer Science (IJEECS), vol. 19, no. 3, pp. 1469-1479, 2020, doi: 10.11591/ijeecs.v19.i3.pp1469-1479. 